Canad. Math. Bull. Vol. 47 (1), 2004 pp. 108-118

\title{
On Universal Schauder Bases in Non-Archimedean Fréchet Spaces
}

\author{
Wiesław Śliwa
}

\begin{abstract}
It is known that any non-archimedean Fréchet space of countable type is isomorphic to a subspace of $c_{0}^{\mathbb{N}}$. In this paper we prove that there exists a non-archimedean Fréchet space $U$ with a basis $\left(u_{n}\right)$ such that any basis $\left(x_{n}\right)$ in a non-archimedean Fréchet space $X$ is equivalent to a subbasis $\left(u_{k_{n}}\right)$ of $\left(u_{n}\right)$. Then any non-archimedean Fréchet space with a basis is isomorphic to a complemented subspace of $U$. In contrast to this, we show that a non-archimedean Fréchet space $X$ with a basis $\left(x_{n}\right)$ is isomorphic to a complemented subspace of $c_{0}^{\mathbb{N}}$ if and only if $X$ is isomorphic to one of the following spaces: $c_{0}, c_{0} \times \mathbb{K}^{\mathbb{N}}, \mathbb{K}^{\mathbb{N}}, c_{0}^{\mathbb{N}}$. Finally, we prove that there is no nuclear non-archimedean Fréchet space $H$ with a basis $\left(h_{n}\right)$ such that any basis $\left(y_{n}\right)$ in a nuclear non-archimedean Fréchet space $Y$ is equivalent to a subbasis $\left(h_{k_{n}}\right)$ of $\left(h_{n}\right)$.
\end{abstract}

\section{Introduction}

In this paper all linear spaces are over a non-archimedean non-trivially valued field $\mathbb{K}$ which is complete under the metric induced by the valuation $|\cdot|: \mathbb{K} \rightarrow[0, \infty)$. For fundamentals of locally convex Hausdorff spaces (lcs) and normed spaces we refer to [5], [7] and [6]. Bases in locally convex spaces are studied in [1]-[4].

Any infinite-dimensional Banach space $E$ of countable type is isomorphic to the Banach space $c_{0}$ of all sequences in $\mathbb{K}$ converging to zero (with the sup-norm $\left.\|\cdot\|\right)([6]$, Theorem 3.16), so $E$ has a basis which is equivalent to the coordinate basis in $c_{0}$.

There exist Fréchet spaces of countable type without bases (see [9, Theorem 3]). However, any infinite-dimensional Fréchet space $E$ contains a closed subspace $X$ with a basis $\left(x_{n}\right)$ (see [8, Theorem 2]). Moreover, any infinite-dimensional Fréchet space $G$ of finite type is isomorphic to the Fréchet space $\mathbb{K}^{\mathbb{N}}$ of all sequences in $\mathbb{K}$ with the topology of pointwise convergence (see [3, Theorem 3.5]), so $G$ has a basis which is equivalent to the coordinate basis in $\mathbb{K}^{\mathrm{N}}$.

Let $\mathcal{F}$ be the family of all bases in Fréchet spaces and let $\mathcal{F}_{0} \subset \mathcal{F}$. A basis $\left(u_{n}\right)$ is universal (respectively quasi-universal) for $\mathcal{F}_{0}$ if $\left(u_{n}\right) \in \mathcal{F}_{0}$ and any basis $\left(x_{n}\right) \in \mathcal{F}_{0}$ is equivalent (respectively quasi-equivalent) to a subbasis $\left(u_{k_{n}}\right)$ of $\left(u_{n}\right)$.

In this paper we study the existence and the uniqueness of universal bases for some important subfamilies of $\mathcal{F}$.

First, we show that there exists a universal basis for the family $\mathcal{F}_{b}$ of all bases in Banach spaces and any two universal bases for $\mathcal{F}_{b}$ are permutatively equivalent (Proposition 1).

\footnotetext{
Received by the editors March 14, 2002; revised April 3, 2002.

AMS subject classification: 46S10, 46A35.

Keywords: universal bases, complemented subspaces with bases.

(C) Canadian Mathematical Society 2004.
} 
Next, we prove that there is a universal basis for the family $\mathcal{F}_{c}$ of all bases in Fréchet spaces with continuous norms and any two universal bases for $\mathcal{F}_{c}$ are permutatively equivalent (Theorem 2). A similar result we also show for the family $\mathcal{F}$ (Theorem 6 ).

It is known that the Fréchet space $c_{0}^{\mathbb{N}}$ is universal for the family of all Fréchet spaces of countable type, that is, $c_{0}^{\mathbb{N}}$ is of countable type and any Fréchet space of countable type is isomorphic to a subspace of $c_{0}^{\mathbb{N}}$ (see [3, Remark 3.6]). We prove that a Fréchet space $E$ with a basis $\left(x_{n}\right)$ is isomorphic to a complemented subspace of $c_{0}^{\mathbb{N}}$ if and only if $E$ is isomorphic to one of the following spaces: $c_{0}, c_{0} \times \mathbb{K}^{\mathbb{N}}, \mathbb{K}^{\mathbb{N}}, c_{0}^{\mathbb{N}}$ (Theorem 7). In contrast to this, if $U$ is a Fréchet space with a basis which is universal for $\mathcal{F}$, then any Fréchet space with a basis is isomorphic to a complemented subspace of $U$. It is unknown whether there exists a Fréchet space $F$ of countable type such that any Fréchet space of countable type is isomorphic to a complemented subspace of $F$. By Remark 9 there is a Fréchet space $X$ of countable type that is not isomorphic to any complemented subspace of a Fréchet space with a basis.

Finally, we prove that there exists no quasi-universal basis for the family $\mathcal{F}_{n}$ of all bases in nuclear Fréchet spaces or for the family $\mathcal{F}_{n c}$ of all bases in nuclear Fréchet spaces with continuous norms (Theorem 10). In particular, there is no universal basis for $\mathcal{F}_{n}$ or $\mathcal{F}_{n c}$.

\section{Preliminaries}

We will denote by $\mathbb{N}, Z, Q$ and $\mathbb{R}$ the sets of all positive integers, all integers, all rational numbers and all real numbers, respectively.

The linear span of a subset $A$ of a linear space $E$ is denoted by $\operatorname{lin} A$.

Let $E, F$ be locally convex spaces. A map $T: E \rightarrow F$ is called a linear homeomorphism if $T$ is linear, one-to-one, surjective and the maps $T, T^{-1}$ are continuous. $E$ is isomorphic to $F(E \simeq F)$ if there exists a linear homeomorphism $T: E \rightarrow F$.

A Fréchet space is a metrizable complete lcs. A Banach space is a normable Fréchet space. Every $n$-dimensional lcs is isomorphic to the Banach space $\mathbb{K}^{n}$.

Let $\left(x_{n}\right)$ be a sequence in a Fréchet space $E$. The series $\sum_{n=1}^{\infty} x_{n}$ is convergent in $E$ if and only if $\lim x_{n}=0$.

Let $\left(x_{n}\right)$ and $\left(y_{n}\right)$ be sequences in locally convex spaces $E$ and $F$, respectively. We say that:

$\left(x_{n}\right)$ is equivalent to $\left(y_{n}\right)$ if there is a linear homeomorphism $T: \operatorname{lin}\left(x_{n}\right) \rightarrow$ $\operatorname{lin}\left(y_{n}\right)$ such that $T x_{n}=y_{n}$ for all $n \in \mathbb{N} ;\left(x_{n}\right)$ is permutatively equivalent to $\left(y_{n}\right)$ if $\left(x_{n}\right)$ is equivalent to a permutation $\left(y_{\pi(n)}\right)$ of $\left(y_{n}\right) ;\left(x_{n}\right)$ is semi equivalent to $\left(y_{n}\right)$ if $\left(x_{n}\right)$ is equivalent to $\left(\alpha_{n} y_{n}\right)$ for some $\left(\alpha_{n}\right) \subset(\mathbb{K} \backslash\{0\}) ;\left(x_{n}\right)$ is quasiequivalent to $\left(y_{n}\right)$ if $\left(x_{n}\right)$ is semi equivalent to a permutation $\left(y_{\pi(n)}\right)$ of $\left(y_{n}\right)$.

A sequence $\left(x_{n}\right)$ in a Fréchet space $E$ is equivalent to a sequence $\left(y_{n}\right)$ in a Fréchet space $F$ if and only if there is a linear homeomorphism $T$ between the closed linear spans of $\left(x_{n}\right)$ and $\left(y_{n}\right)$ such that $T x_{n}=y_{n}, n \in \mathbb{N}$.

A sequence $\left(x_{n}\right)$ in a lcs $E$ is a basis in $E$ if each $x \in E$ can be written uniquely as $x=\sum_{n=1}^{\infty} \alpha_{n} x_{n}$ with $\left(\alpha_{n}\right) \subset \mathbb{K}$. If additionally the coefficient functionals $f_{n}: E \rightarrow \mathbb{K}$, $x \rightarrow \alpha_{n}(n \in \mathbb{N})$ are continuous, then $\left(x_{n}\right)$ is a Schauder basis in $E$.

A subsequence $\left(x_{k_{n}}\right)$ of a basis $\left(x_{n}\right)$ in a lcs $E$ is a subbasis of $\left(x_{n}\right)$. 
As in the real or complex case any basis in a Fréchet space is a Schauder basis (see [4, Corollary 4.2]).

By a seminorm on a linear space $E$ we mean a function $p: E \rightarrow[0, \infty)$ such that $p(\alpha x)=|\alpha| p(x)$ for all $\alpha \in \mathbb{K}, x \in E$ and $p(x+y) \leq \max \{p(x), p(y)\}$ for all $x, y \in E$. A seminorm $p$ on $E$ is a norm if $\operatorname{ker} p:=\{x \in E: p(x)=0\}=\{0\}$.

Two norms $p, q$ on a linear space $E$ are equivalent if there exist positive numbers $a, b$ such that $a p(x) \leq q(x) \leq b p(x)$ for each $x \in E$. Every two norms on a finitedimensional linear space are equivalent.

The set of all continuous seminorms on a metrizable lcs $E$ is denoted by $\mathcal{P}(E)$. A non-decreasing sequence $\left(p_{k}\right) \subset \mathcal{P}(E)$ is a base in $\mathcal{P}(E)$ if for every $p \in \mathcal{P}(E)$ there exists $k \in \mathbb{N}$ with $p \leq p_{k}$. A sequence $\left(p_{k}\right)$ of norms on $E$ is a base of norms in $\mathcal{P}(E)$ if it is a base in $\mathcal{P}(E)$.

Any metrizable lcs $E$ possesses a base $\left(p_{k}\right)$ in $\mathcal{P}(E)$. Every metrizable lcs $E$ with a continuous norm has a base of norms $\left(p_{k}\right)$ in $\mathcal{P}(E)$.

A metrizable lcs $E$ is of finite type if $\operatorname{dim}(E / \operatorname{ker} p)<\infty$ for any $p \in \mathcal{P}(E)$, and of countable type if $E$ contains a linearly dense countable set.

Let $p$ be a seminorm on a linear space $E$. A sequence $\left(x_{n}\right) \subset E$ is 1-orthogonal with respect to $p$ if $p\left(\sum_{i=1}^{n} \alpha_{i} x_{i}\right)=\max _{1 \leq i \leq n} p\left(\alpha_{i} x_{i}\right)$ for all $n \in \mathbb{N}, \alpha_{1}, \ldots, \alpha_{n} \in \mathbb{K}$.

A sequence $\left(x_{n}\right)$ in a metrizable lcs $E$ is 1-orthogonal with respect to $\left(p_{k}\right) \subset \mathcal{P}(E)$ if $\left(x_{n}\right)$ is 1 -orthogonal with respect to $p_{k}$ for any $k \in \mathbb{N}$.

A sequence $\left(x_{n}\right)$ in a metrizable lcs $E$ is orthogonal if it is 1-orthogonal with respect to a base $\left(p_{k}\right)$ in $\mathcal{P}(E)$. (In [6] a sequence $\left(x_{n}\right)$ in a normed space $(E,\|\| \cdot \|)$ is called orthogonal if it is 1-orthogonal with respect to the norm $\|\mid \cdot\|$.)

A linearly dense orthogonal sequence of non-zero elements in a metrizable lcs $E$ is an orthogonal basis in $E$.

Every orthogonal basis in a metrizable lcs $E$ is a Schauder basis in $E$ (see [3, Proposition 1.4]) and every Schauder basis in a Fréchet space $F$ is an orthogonal basis in $F$ (see [3, Proposition 1.7]).

Let $B=\left(b_{k, n}\right)$ be an infinite real matrix such that $\forall k, n \in \mathbb{N}: 0<b_{k, n} \leq b_{k+1, n}$.

The space $K(B)=\left\{\left(\alpha_{n}\right) \subset \mathbb{K}: \lim _{n}\left|\alpha_{n}\right| b_{k, n}=0\right.$ for all $\left.k \in \mathbb{N}\right\}$ with the base of norms $\left(p_{k}\right): p_{k}\left(\left(\alpha_{n}\right)\right)=k \max _{n}\left|\alpha_{n}\right| b_{k, n}, k \in \mathbb{N}$, is called the Köthe space associated with the matrix $B . K(B)$ is a Fréchet space and the sequence $\left(e_{n}\right)$ of coordinate vectors forms a basis in it (see [2, Proposition 2.2]). The coordinate basis $\left(e_{n}\right)$ is 1-orthogonal with respect to the base of norms $\left(p_{k}\right)$.

Let $E$ be a Fréchet space with a Schauder basis $\left(x_{n}\right)$ which is 1-orthogonal with respect to a base of norms $\left(p_{k}\right)$ in $\mathcal{P}(E)$. Then $E$ is nuclear if and only if

$$
\forall k \in \mathbb{N} \exists m>k: \lim _{n}\left[p_{k}\left(x_{n}\right) / p_{m}\left(x_{n}\right)\right]=0
$$

(see [2, Propositions 2.4 and 3.5]).

\section{Results}

We start with the following proposition. 
Proposition 1 There exists a universal basis $\left(w_{n}\right)$ for the family $\mathcal{F}_{b}$ of all bases in Banach spaces. A basis $\left(x_{n}\right) \in \mathcal{F}_{b}$ is universal for $\mathcal{F}_{b}$ if and only if it is permutatively equivalent to $\left(w_{n}\right)$.

Proof It is easy to check that an orthogonal sequence $\left(x_{n}\right)$ in a Banach space $\left(X,\||\cdot|\|_{X}\right)$ is equivalent to an orthogonal sequence $\left(y_{n}\right)$ in a Banach space $\left(Y,\|\| \cdot \|_{Y}\right)$ if and only if there exists a number $A \geq 1$ such that

$$
\forall n \in \mathbb{N}: A^{-1}\left\|x_{n}\right\|_{X} \leq\|\| y_{n}\left\|_{Y} \leq A \mid\right\| x_{n} \|_{X}
$$

Let $\left\{N_{t}: t \in Z\right\}$ be a family of pairwise disjoint infinite subsets of $\mathbb{N}$ such that $\bigcup\left\{N_{t}: t \in Z\right\}=\mathbb{N}$. Let $\alpha \in \mathbb{K}$ with $|\alpha|>1$. Denote by $\left(e_{n}\right)$ the coordinate basis in $c_{0}$. Put $w_{n}=\alpha^{t} e_{n}$ for all $n \in N_{t}, t \in Z$. Clearly, $\left(w_{n}\right)$ is a basis in $c_{0}$.

Let $\pi$ be a permutation of $\mathbb{N}$ and let $\left(y_{n}\right)$ be a basis in a Banach space $(Y,\|\cdot\|)$. We can choose an increasing sequence $\left(k_{n}\right) \subset \mathbb{N}$ such that

$$
\forall n \in \mathbb{N} \forall t \in Z:\left[k_{n} \in \pi^{-1}\left(N_{t}\right) \Leftrightarrow|\alpha|^{t} \leq\left.\left\|\left|y_{n}\right|\right\||<| \alpha\right|^{t+1}\right] .
$$

Then $\forall n \in \mathbb{N}:\left\|w_{\pi\left(k_{n}\right)}\right\| \leq\left\|y_{n}\left|\|<|\alpha|\| w_{\pi\left(k_{n}\right)} \|\right.\right.$. It follows that $\left(y_{n}\right)$ is equivalent to $\left(w_{\pi\left(k_{n}\right)}\right)$. Thus for any permutation $\pi$ of $\mathbb{N}$ the basis $\left(w_{\pi(n)}\right)$ in $c_{0}$ is universal for $\mathcal{F}_{b}$. Hence any basis $\left(x_{n}\right) \in \mathcal{F}_{b}$ which is permutatively equivalent to $\left(w_{n}\right)$ is universal for $\mathcal{F}_{b}$.

Now, let us assume that a basis $\left(x_{n}\right)$ in a Banach space $(X,\||\cdot|\|)$ is universal for $\mathcal{F}_{b}$. Then the basis $\left(w_{n}\right)$ is equivalent to a subbasis $\left(x_{s_{n}}\right)$ of $\left(x_{n}\right)$. Thus there exists $t_{0} \in \mathbb{N}$ such that

$$
\forall n \in \mathbb{N}:|\alpha|^{-t_{0}}\left\|w_{n}\right\| \leq\left\|\left|x_{s_{n}}\left\|\left.|<| \alpha\right|^{t_{0}}\right\| w_{n} \| .\right.\right.
$$

Hence $\forall t \in Z \forall n \in N_{t}:|\alpha|^{t-t_{0}} \leq \||| x_{s_{n}}||<|\alpha|^{t+t_{0}}$. Therefore for any $t \in Z$ the set $M_{t}=\left\{n \in \mathbb{N}:|\alpha|^{2 t_{0} t} \leq\left.\left\||| x_{n}\right\||<| \alpha\right|^{2 t_{0}(t+1)}\right\}$ is infinite. Let $\pi$ be a permutation of $\mathbb{N}$ such that

$$
\pi\left(M_{t}\right)=\left\{n \in \mathbb{N}:|\alpha|^{2 t_{0} t} \leq\left\|w_{n}\right\|<|\alpha|^{2 t_{0}(t+1)}\right\}, \quad t \in Z
$$

Then $\forall n \in \mathbb{N}:|\alpha|^{-2 t_{0}}\left\|\left|x_{n}\right|\right\|<\left\|w_{\pi(n)}\right\|<|\alpha|^{2 t_{0}}\left\|\left|x_{n} \|\right| \mid\right.$, so $\left(x_{n}\right)$ is equivalent to $\left(w_{\pi(n)}\right)$. Thus $\left(x_{n}\right)$ is permutatively equivalent to $\left(w_{n}\right)$.

Now, we prove the following.

Theorem 2 There exists a universal basis $\left(v_{n}\right)$ for the family $\mathcal{F}_{c}$ of all bases in Fréchet spaces with continuous norms. A basis $\left(x_{n}\right) \in \mathcal{F}_{c}$ is universal (respectively quasiuniversal) for $\mathcal{F}_{c}$ if and only if $\left(x_{n}\right)$ is permutatively equivalent (respectively quasiequivalent) to $\left(v_{n}\right)$.

Proof For any $m \in \mathbb{N}$ the set $A_{m}=\left\{\left(x_{1}, \ldots, x_{m}\right) \in \mathbb{R}^{m}: 0=x_{0}<\cdots<x_{m}\right\}$ is open in $\mathbb{R}^{m}$. Moreover, $\bigcup_{m=1}^{\infty} A_{m} \cap Q^{m}=\left\{d_{n}: n \in \mathbb{N}\right\}$ for some sequence $\left(d_{n}\right)$. Assume that $d_{n} \in Q^{m(n)}, n \in \mathbb{N}$. For every $n \in \mathbb{N}$ there exists an increasing sequence $\left(b_{k, n}^{0}\right)_{k=1}^{\infty} \subset Q$ such that $\left(b_{1, n}^{0}, \ldots, b_{m(n), n}^{0}\right)=d_{n}$. Put $B=\left(b_{k, n}^{0}\right)$. Let 
$\left(v_{n}\right)$ be the coordinate basis in the Köthe space $V=K(B)$. For any $m \in \mathbb{N}$ the set $D_{m}=\left\{\left(b_{1, n}^{0}, \ldots, b_{m, n}^{0}\right): n \in \mathbb{N}\right\}$ is dense in $A_{m}$, since $D_{m}=A_{m} \cap Q^{m}$.

Let $F$ be a Fréchet space with a continuous norm and with a basis $\left(y_{n}\right)$. Then $\left(y_{n}\right)$ is 1-orthogonal with respect to a base of norms $\left(p_{k}\right)$ in $\mathcal{P}(F)$. Put $b_{k, n}=p_{k}\left(y_{n}\right)$ for $k, n \in \mathbb{N}$. For every $m \in \mathbb{N}$ the set $S_{m}=A_{m} \cap \prod_{k=1}^{m}\left(b_{k, m}, 2 b_{k, m}\right)$ is open in $A_{m}$. Moreover, $S_{m} \neq \varnothing$, since $\left(t b_{1, m}, \ldots, t^{m} b_{m, m}\right) \in S_{m}$ for any $t \in\left(1,2^{1 /(m+1)}\right)$. Hence $D_{m} \cap S_{m}$ is a dense subset of $S_{m}$ for all $m \in \mathbb{N}$.

Let $\pi$ be a permutation of $\mathbb{N}$ (and $\left(c_{n}\right) \subset \mathbb{N}$ ). Then there exists an increasing sequence $\left(k_{n}\right) \subset \mathbb{N}$ (with $k_{n+1}>c_{k_{n}}, n \in \mathbb{N}$ ) such that $\left(b_{1, \pi\left(k_{n}\right)}^{0}, \ldots, b_{n, \pi\left(k_{n}\right)}^{0}\right) \in S_{n}$, $n \in \mathbb{N}$.

Then $\forall n \in \mathbb{N} \forall 1 \leq i \leq n: b_{i, n}<b_{i, \pi\left(k_{n}\right)}^{0}<2 b_{i, n}$. Hence we get $a_{j}:=$ $\sup _{n}\left(b_{j, n} / b_{j, \pi\left(k_{n}\right)}^{0}\right)<\infty$ and $b_{j}:=\sup _{n}\left(b_{j, \pi\left(k_{n}\right)}^{0} / b_{j, n}\right)<\infty$ for any $j \in \mathbb{N}$.

It follows that the basis $\left(y_{n}\right)$ is equivalent to the subbasis $\left(v_{\pi\left(k_{n}\right)}\right)$ of $\left(v_{\pi(n)}\right)$. Indeed, let $T: \operatorname{lin}\left(y_{n}\right) \rightarrow \operatorname{lin}\left(v_{\pi\left(k_{n}\right)}\right)$ be a linear map with $T y_{n}=v_{\pi\left(k_{n}\right)}, n \in \mathbb{N}$. Clearly, $T$ is a bijection. Let $\left(q_{j}\right)$ be the standard base of norms in $\mathcal{P}(V)$. Let $j \in \mathbb{N}$ and $y \in \operatorname{lin}\left(y_{n}\right)$. Then $y=\sum_{i=1}^{m} \alpha_{i} y_{i}$ for some $m \in \mathbb{N}, \alpha_{1}, \ldots, \alpha_{m} \in \mathbb{K}$. We have

$$
\begin{aligned}
& p_{j}(y)=\max _{1 \leq i \leq m}\left|\alpha_{i}\right| p_{j}\left(y_{i}\right) \leq a_{j} \max _{1 \leq i \leq m}\left|\alpha_{i}\right| q_{j}\left(v_{\pi\left(k_{i}\right)}\right)=a_{j} q_{j}(T y), \\
& q_{j}(T y)=\max _{1 \leq i \leq m}\left|\alpha_{i}\right| q_{j}\left(v_{\pi\left(k_{i}\right)}\right) \leq b_{j} \max _{1 \leq i \leq m}\left|\alpha_{i}\right| p_{j}\left(y_{i}\right)=b_{j} p_{j}(y) .
\end{aligned}
$$

Therefore $T$ is a linear homeomorphism.

Thus for any permutation $\pi$ of $\mathbb{N}$ the basis $\left(v_{\pi(n)}\right)$ in $V$ is universal for $\mathcal{F}_{c}$. Hence any basis $\left(x_{n}\right) \in \mathcal{F}_{c}$ which is permutatively equivalent (respectively quasi-equivalent) to $\left(v_{n}\right)$ is universal (respectively quasi-universal) for $\mathcal{F}_{c}$.

Now, let us assume that a basis $\left(x_{n}\right)$ in a Fréchet space $X$ with a continuous norm is universal for $\mathcal{F}_{c}$. We shall show that $\left(x_{n}\right)$ is permutatively equivalent to $\left(v_{n}\right)$.

Suppose that $\left(x_{n}\right)$ is 1-orthogonal with respect to a base of norms $\left(r_{k}\right)$ in $\mathcal{P}(X)$. The basis $\left(v_{n}\right)$ is equivalent to a subbasis $\left(x_{k_{n}}\right)$ of $\left(x_{n}\right)$. Put $M=\left\{k_{n}: n \in \mathbb{N}\right\}$ and $L=(\mathbb{N} \backslash M)$. Clearly, it is enough to consider the case when $L \neq \varnothing$.

Denote by $G$ the closed linear span of $\left\{x_{n}: n \in L\right\}$. It is easy to show that the linear space $c_{0}(G)=\left\{\left(y_{n}\right) \subset G: \lim y_{n}=0\right\}$ with the base of norms $\left(r_{k}^{0}\right): r_{k}^{0}\left(\left(y_{n}\right)\right)=$ $\max _{n} r_{k}\left(y_{n}\right), k \in \mathbb{N}$ is a Fréchet space.

For an infinite countable set $A$ we will denote by $S(A)$ an arbitrary sequence $\left(a_{n}\right)$ with $\left\{a_{n}: n \in \mathbb{N}\right\}=A$ such that $a_{n} \neq a_{m}$ for all $n, m \in \mathbb{N}$ with $n \neq m$.

For $i, j \in \mathbb{N}, n \in L$ put $x_{i, j}^{n}=0$ if $j \neq i$, and $x_{i, j}^{n}=x_{n}$ if $j=i$. Let $x_{i}^{n}=\left(x_{i, j}^{n}\right)_{j=1}^{\infty}$ for all $i \in \mathbb{N}, n \in L$. Set $\left(s_{m}\right)=S\left(\left\{x_{i}^{n}: i \in \mathbb{N}, n \in L\right\}\right)$. It is easy to check that $\left(s_{m}\right)$ is a basis in the Fréchet space $c_{0}(G)$ which is permutatively equivalent to the basis $S\left(\left\{\left(x_{n}, 0\right) ; n \in L\right\} \cup\left\{\left(0, s_{n}\right): n \in \mathbb{N}\right\}\right)$ in $G \times c_{0}(G)$.

The basis $\left(s_{n}\right)$ is equivalent to a subbasis $\left(v_{m_{n}}\right)$ of $\left(v_{n}\right)$. Hence $\left(s_{n}\right)$ is equivalent to $\left(x_{k_{m_{n}}}\right)$. Put $W=\left\{k_{m_{n}}: n \in \mathbb{N}\right\}$ and $K=(M \backslash W)$. Since $W=[\mathbb{N} \backslash(L \cup K)]$, then $\left(x_{n}\right)$ is permutatively equivalent to the basis $S\left(\left\{\left(x_{n}, 0,0\right): n \in L\right\} \cup\left\{\left(0, s_{n}, 0\right)\right.\right.$ : $\left.n \in \mathbb{N}\} \cup\left\{\left(0,0, x_{n}\right): n \in K\right\}\right)$ in $G \times c_{0}(G) \times H$, where $H$ is the closed linear span of $\left\{x_{n}: n \in K\right\}$. But $S\left(\left\{\left(x_{n}, 0\right): n \in L\right\} \cup\left\{\left(0, s_{n}\right): n \in \mathbb{N}\right\}\right)$ is permutatively equivalent 
to $\left(x_{k_{m_{n}}}\right)$, so $\left(x_{n}\right)$ is permutatively equivalent to $S\left(\left\{\left(x_{n}, 0\right): n \in W\right\} \cup\left\{\left(0, x_{n}\right): n \in\right.\right.$ $K\})$. Thus $\left(x_{n}\right)$ is permutatively equivalent to $S\left(\left\{x_{n}: n \in M\right\}\right)$. Hence $\left(x_{n}\right)$ is permutatively equivalent to $\left(v_{n}\right)$.

Similarly, one can show the following. If a basis $\left(x_{n}\right)$ in a Fréchet space $X$ with a continuous norm is quasi-universal for $\mathcal{F}_{c}$, then $\left(x_{n}\right)$ is quasi-equivalent to $\left(v_{n}\right)$. (In this case $\left(v_{n}\right)$ is quasi-equivalent to a subbasis $\left(x_{k_{n}}\right)$ of $\left(x_{n}\right)$ and $\left(s_{n}\right)$ is semi equivalent to $\left(x_{k_{\pi\left(m_{n}\right)}}\right)$ for some increasing sequence $\left(m_{n}\right) \subset \mathbb{N}$ and some permutation $\pi$ of $\mathbb{N}$; instead of $W$ we take $W^{\prime}=\left\{k_{\pi\left(m_{n}\right)}: n \in \mathbb{N}\right\}$.)

From now on, $\left(v_{n}\right)$ is a universal basis for $\mathcal{F}_{c}$ and $V$ is a Fréchet space with the basis $\left(v_{n}\right)$.

By the proof of Theorem 2 we obtain

Remark 3 For any $\left(z_{n}\right) \in \mathcal{F}_{c}$ and any sequence $\left(c_{n}\right) \subset \mathbb{N}$ there exists an increasing sequence $\left(k_{n}\right) \subset \mathbb{N}$ with $k_{n+1}>c_{k_{n}}, n \in \mathbb{N}$ such that $\left(z_{n}\right)$ is equivalent to $\left(v_{k_{n}}\right)$.

Clearly, any Fréchet space with a continuous norm and with a basis is isomorphic to a complemented subspace of $V$. The following is also true.

Proposition 4 Let E be a Fréchet space with a continuous norm and with a basis. If any Fréchet space with a continuous norm and with a basis is isomorphic to a complemented subspace of $E$, then $E$ is isomorphic to $V$.

Proof It is clear that the Fréchet spaces $c_{0}(E)$ and $c_{0}(V)$ have continuous norms and bases (see the proof of Theorem 2). Moreover, $E \times c_{0}(E)$ and $V \times c_{0}(V)$ are isomorphic to $c_{0}(E)$ and $c_{0}(V)$, respectively. Thus there exist Fréchet spaces $G$ and $H$ such that $V \simeq c_{0}(E) \times H \simeq E \times c_{0}(E) \times H \simeq E \times V$ and $E \simeq c_{0}(V) \times G \simeq V \times c_{0}(V) \times G \simeq V \times E$. Hence $E$ is isomorphic to $V$.

By the closed graph theorem (see [5, Theorem 2.49]) we get

Remark 5 Let $\left(x_{n}\right)$ be a basis in a Fréchet space $E$ and $\left(y_{n}\right)$ a basis in a Fréchet space $F$. Then the following conditions are equivalent:

(1) $\left(x_{n}\right)$ is equivalent to $\left(y_{n}\right)$;

(2) for any $\left(\beta_{n}\right) \subset \mathbb{K}$ the sequence $\left(\beta_{n} x_{n}\right)$ is convergent to 0 in $E$ if and only if the sequence $\left(\beta_{n} y_{n}\right)$ is convergent to 0 in $F$;

(3) for any $\left(\beta_{n}\right) \subset \mathbb{K}$ the series $\sum_{n=1}^{\infty} \beta_{n} x_{n}$ is convergent in $E$ if and only if the series $\sum_{n=1}^{\infty} \beta_{n} y_{n}$ is convergent in $F$.

Using Remark 5 we shall prove the following.

Theorem 6 There exists a universal basis $\left(u_{n}\right)$ for the family $\mathcal{F}$ of all bases in Fréchet spaces. A basis $\left(x_{n}\right) \in \mathcal{F}$ is universal (respectively quasi-universal) for $\mathcal{F}$ if and only if $\left(x_{n}\right)$ is permutatively equivalent (respectively quasi-equivalent) to $\left(u_{n}\right)$. 
Proof Put $U=V^{\mathbb{N}}$. Assume that $\left(v_{n}\right)$ is 1-orthogonal with respect to a base of norms $\left(q_{k}\right)$ in $\mathcal{P}(V)$. Set $p_{k}\left(\left(x_{n}\right)\right)=k \max _{1 \leq n \leq k} q_{k}\left(x_{n}\right)$ for $\left(x_{n}\right) \in U, k \in \mathbb{N}$. Clearly, $\left(p_{k}\right)$ is a base in $\mathcal{P}(U)$. For $n, i, j \in \mathbb{N}$ we put $v_{i, j}^{n}=0$ if $j \neq i$, and $v_{i, j}^{n}=v_{n}$ if $j=i$. Let $v_{i}^{n}=\left(v_{i, j}^{n}\right)_{j=1}^{\infty}$ for all $n, i \in \mathbb{N}$. It is easy to see that there exists a bijection $\varphi: \mathbb{N} \times \mathbb{N} \rightarrow \mathbb{N}$ such that the sequence $(\varphi(i, n))_{n=1}^{\infty}$ is increasing for any $i \in \mathbb{N}$. Let $u_{\varphi(i, n)}=v_{i}^{n}$ for $i, n \in \mathbb{N}$. Of course, $\left(u_{m}\right)$ is a basis in $U$. Moreover, for any $i \in \mathbb{N}$ the subbasis $\left(u_{\varphi(i, n)}\right)_{n=1}^{\infty}$ of $\left(u_{m}\right)$ is equivalent to $\left(v_{n}\right)$, so it is a universal basis for $\mathcal{F}_{c}$. Put $M_{i}=\{\varphi(i, n): n \in \mathbb{N}\}, i \in \mathbb{N}$. Clearly, $M_{i}=\left\{m \in \mathbb{N}: u_{m} \in\left(\operatorname{ker} p_{i-1} \backslash \operatorname{ker} p_{i}\right)\right\}$ for $i \in \mathbb{N}$, where $p_{0}(x)=0$ for $x \in U$.

Now, let us assume that $\left(y_{n}\right)$ is a basis in a Fréchet space $Y$. Then $\left(y_{n}\right)$ is 1orthogonal with respect to a base $\left(r_{k}\right)$ in $\mathcal{P}(Y)$. Let $r_{0}(y)=0$ for $y \in Y$. Put $N_{i}=\left\{n \in \mathbb{N}: y_{n} \in\left(\operatorname{ker} r_{i-1} \backslash \operatorname{ker} r_{i}\right)\right\}$ for $i \in \mathbb{N}$. Denote by $W$ the set of all $i \in \mathbb{N}$ for which the set $N_{i}$ is infinite. For any $i \in W$ the sequence $\left(y_{m}\right)_{m \in N_{i}}$ is a basis in the closed linear span $Y_{i}$ of $\left\{y_{m}: m \in N_{i}\right\}$ and $r_{i} \mid Y_{i}$ is a continuous norm on $Y_{i}$.

Let $\pi$ be a permutation of $\mathbb{N}$. By the proof of Theorem 2 we can construct inductively an increasing sequence $\left(k_{n}\right) \subset \mathbb{N}$ with $\left\{\pi\left(k_{n}\right): n \in N_{i}\right\} \subset M_{i}, i \in \mathbb{N}$, such that $\left(y_{n}\right)_{n \in N_{i}}$ is equivalent to $\left(u_{\pi\left(k_{n}\right)}\right)_{n \in N_{i}}$ for any $i \in W$. We shall prove that $\left(y_{n}\right)$ is equivalent to $\left(u_{\pi\left(k_{n}\right)}\right)$.

Let $\left(\beta_{n}\right) \subset \mathbb{K}$. Assume that $\lim _{n} \beta_{n} y_{n}=0$. Then $\lim _{n \in N_{i}} \beta_{n} y_{n}=0$ for any $i \in W$. By Remark $5, \lim _{n \in N_{i}} \beta_{n} u_{\pi\left(k_{n}\right)}=0$ for any $i \in W$. We show that $\lim _{n} \beta_{n} u_{\pi\left(k_{n}\right)}=0$. Suppose, by contradiction, that there exists a neighborhood $M$ of 0 in $U$ and an increasing sequence $\left(d_{m}\right) \subset \mathbb{N}$ such that $\left(\beta_{d_{m}} u_{\pi\left(k_{d_{m}}\right)}\right) \subset(U \backslash M)$. Then for any $i \in \mathbb{N}$ the set $\left\{m \in \mathbb{N}: d_{m} \in N_{i}\right\}$ is finite. Therefore for every $i \in \mathbb{N}$ there is $m_{i} \in \mathbb{N}$ with $\left(d_{m}\right)_{m=m_{i}}^{\infty} \subset \bigcup_{j=i+1}^{\infty} N_{j}$. Hence $\left(\pi\left(k_{d_{m}}\right)\right)_{m=m_{i}}^{\infty} \subset \bigcup_{j=i+1}^{\infty} M_{j}$, so $p_{i}\left(\beta_{d_{m}} u_{\pi\left(k_{d_{m}}\right)}\right)=0$ for all $m, i \in \mathbb{N}$ with $m \geq m_{i}$. It follows that $\lim _{m} \beta_{d_{m}} u_{\pi\left(k_{d_{m}}\right)}=0$, a contradiction. Thus $\lim _{n} \beta_{n} u_{\pi\left(k_{n}\right)}=0$.

Similarly, assuming that $\lim _{n} \beta_{n} u_{\pi\left(k_{n}\right)}=0$ we get $\lim _{n} \beta_{n} y_{n}=0$. By Remark 5, $\left(y_{n}\right)$ is equivalent to $\left(u_{\pi\left(k_{n}\right)}\right)$. Thus the basis $\left(u_{\pi(n)}\right)$ in $U$ is universal for $\mathcal{F}$. Hence any basis $\left(x_{n}\right) \in \mathcal{F}$, which is permutatively equivalent (respectively quasi-equivalent) to $\left(u_{n}\right)$, is universal (respectively quasi-universal) for $\mathcal{F}$.

Now, let us assume that a basis $\left(x_{n}\right)$ in a Fréchet space $X$ is universal (respectively quasi-universal) for $\mathcal{F}$. As in the proof of Theorem 2 one can show that $\left(x_{n}\right)$ is permutatively equivalent (respectively quasi-equivalent) to $\left(u_{n}\right)$.

The Fréchet spaces $c_{0}^{\mathbb{N}}$ and $V^{\mathbb{N}}$ are universal for the family of all Fréchet spaces of countable type. By Theorem 6 any Fréchet space with a basis is isomorphic to a complemented subspace of $V^{\mathbb{N}}$. In contrast to this, we shall prove the following result for $c_{0}^{\mathbb{N}}$.

Theorem 7 A Fréchet space $X$ with a basis $\left(x_{n}\right)$ is isomorphic to a complemented subspace of $c_{0}^{\mathbb{N}}$ if and only if $X$ is isomorphic to one of the following spaces: $c_{0}, c_{0} \times \mathbb{K}^{\mathbb{N}}$, $\mathbb{K}^{\mathbb{N}}, c_{0}^{\mathbb{N}}$.

Proof Clearly, any Fréchet space, which is isomorphic to one of the following spaces: $c_{0}, c_{0} \times \mathbb{K}^{\mathbb{N}}, \mathbb{K}^{\mathbb{N}}, c_{0}^{\mathbb{N}}$, is isomorphic to a complemented subspace of $c_{0}^{\mathbb{N}}$. 
To prove the converse, let us denote by $P$ a linear continuous projection from $c_{0}^{\mathbb{N}}$ onto a complemented subspace $Y$ of $c_{0}^{\mathbb{N}}$ which is isomorphic to $X$. Let $\left(e_{n}\right)$ be the coordinate basis in $c_{0}$. For $n, i, j \in \mathbb{N}$ we put $e_{i, j}^{n}=0$ if $j \neq i$, and $e_{i, j}^{n}=e_{n}$ if $j=i$. Set $e_{i}^{n}=\left(e_{i, j}^{n}\right)_{j=1}^{\infty}$ for $n, i \in \mathbb{N}$. Let $\varphi: \mathbb{N} \times \mathbb{N} \rightarrow \mathbb{N}$ be a bijection. Put $z_{\varphi(k, n)}=e_{k}^{n}$ for all $n, k \in \mathbb{N}$. Clearly, $\left(z_{n}\right)$ is a basis in $c_{0}^{\mathbb{N}}$. Let $\left(y_{n}\right)$ be a basis in $Y$. Denote by $\left(f_{n}\right)$ and $\left(h_{n}\right)$ the sequences of coefficient functionals associated with the bases $\left(z_{n}\right)$ and $\left(y_{n}\right)$, respectively. Put $g_{n}(z)=h_{n}(P z)$ for $n \in \mathbb{N}$ and $z \in c_{0}^{\mathbb{N}}$. Since

$$
\begin{aligned}
1 & =\left|g_{n}\left(y_{n}\right)\right|=\left|g_{n}\left(\sum_{k=1}^{\infty} f_{k}\left(y_{n}\right) z_{k}\right)\right| \\
& =\left|\sum_{k=1}^{\infty} f_{k}\left(y_{n}\right) g_{n}\left(z_{k}\right)\right| \leq \max _{k}\left|f_{k}\left(y_{n}\right) g_{n}\left(z_{k}\right)\right|, \quad n \in \mathbb{N},
\end{aligned}
$$

then for any $n \in \mathbb{N}$ there exists $t_{n} \in \mathbb{N}$ with $\left|f_{t_{n}}\left(y_{n}\right) g_{n}\left(z_{t_{n}}\right)\right| \geq 1$.

Put $p_{k}\left(\left(\alpha_{n}\right)\right)=k \max _{1 \leq n \leq k}\left\|\alpha_{n}\right\|$ for all $k \in \mathbb{N}$ and $\left(\alpha_{n}\right) \in c_{0}^{\mathbb{N}}$. Clearly, $\left(p_{k}\right)$ is a base in $\mathcal{P}\left(c_{0}^{\mathbb{N}}\right)$ and the basis $\left(z_{n}\right)$ is 1-orthogonal with respect to the base $\left(p_{k}\right)$.

For any $k \in \mathbb{N}$ there exist $q_{k} \in \mathcal{P}\left(c_{0}^{\mathbb{N}}\right)$ and $s_{k} \in \mathbb{N}$ with $p_{k} \leq q_{k} \leq p_{s_{k}}$ and $q_{k} \circ P \leq p_{s_{k}}$ such that $\left(y_{n}\right)$ is 1 -orthogonal with respect to $q_{k}$. For all $n, k \in \mathbb{N}$ we obtain

$$
\begin{aligned}
p_{k}\left(f_{t_{n}}\left(y_{n}\right) z_{t_{n}}\right) & \leq \max _{m} p_{k}\left(f_{m}\left(y_{n}\right) z_{m}\right)=p_{k}\left(y_{n}\right) \leq\left|g_{n}\left(z_{t_{n}}\right)\right|^{-1} \max _{m} q_{k}\left(g_{m}\left(z_{t_{n}}\right) y_{m}\right) \\
& =\left|g_{n}\left(z_{t_{n}}\right)\right|^{-1} q_{k}\left(P z_{t_{n}}\right) \leq p_{s_{k}}\left(f_{t_{n}}\left(y_{n}\right) z_{t_{n}}\right) .
\end{aligned}
$$

Hence

$$
p_{k}\left(f_{t_{n}}\left(y_{n}\right) z_{t_{n}}\right) \leq p_{k}\left(y_{n}\right) \leq p_{s_{k}}\left(f_{t_{n}}\left(y_{n}\right) z_{t_{n}}\right) \quad \text { for all } k, n \in \mathbb{N} .
$$

Put $r_{k}(y)=\max _{n}\left|h_{n}(y)\right| p_{k}\left(f_{t_{n}}\left(y_{n}\right) z_{t_{n}}\right)$ for $k \in \mathbb{N}, y \in Y$.

By $(*)$, we get $r_{k}(y) \leq \max _{n}\left|h_{n}(y)\right| q_{k}\left(y_{n}\right)=q_{k}(y) \leq p_{s_{k}}(y)$, and $p_{k}(y) \leq$ $\max _{n}\left|h_{n}(y)\right| p_{k}\left(y_{n}\right) \leq \max _{n}\left|h_{n}(y)\right| p_{s_{k}}\left(f_{t_{n}}\left(y_{n}\right) z_{t_{n}}\right)=r_{s_{k}}(y)$.

Thus $\left(r_{k}\right)$ is a base in $\mathcal{P}(Y)$. Put $b_{n}=\left(y_{n} / f_{t_{n}}\left(y_{n}\right)\right), n \in \mathbb{N}$. Clearly, $\left(b_{n}\right)$ is a basis in $Y$ which is 1-orthogonal with respect to $\left(r_{k}\right)$. Let $k, n \in \mathbb{N}$. Since $r_{k}\left(b_{n}\right)=p_{k}\left(z_{t_{n}}\right)$, then $r_{k}\left(b_{n}\right)=0$ or $r_{k}\left(b_{n}\right)=k$. Set $r_{0}(y)=0$ for $y \in Y$.

Put $N_{k}=\left\{n \in \mathbb{N}: b_{n} \in\left(\operatorname{ker} r_{k-1} \backslash \operatorname{ker} r_{k}\right)\right\}, k \in \mathbb{N}$. Clearly, $\bigcup_{k=1}^{\infty} N_{k}=\mathbb{N}$ and $N_{i} \cap N_{j}=\varnothing$ for $i, j \in \mathbb{N}$ with $i \neq j$.

Consider four cases:

Case 1 For some $k_{0} \in \mathbb{N}$ we have $\bigcup_{k=1}^{k_{0}} N_{k}=\mathbb{N}$ : Then $\forall k \geq k_{0} \forall n \in \mathbb{N}: r_{k}\left(b_{n}\right)=k$. Hence $Y$ is normable, so it is isomorphic to $c_{0}$.

Case 2 For any $k \in \mathbb{N}$ the set $N_{k}$ is finite: Let $k \in \mathbb{N}$. Since $\left\{b_{n}: n \in \bigcup_{i=k+1}^{\infty} N_{i}\right\} \subset$ $\operatorname{ker} r_{k}$, then $\operatorname{dim}\left(Y / \operatorname{ker} r_{k}\right)<\infty$. Thus $Y$ is of finite type, so it is isomorphic to $\mathbb{K}^{\mathbb{N}}$.

Case 3 For some increasing sequence $\left(i_{n}\right) \subset \mathbb{N}$ the sets $N_{i_{n}}, n \in \mathbb{N}$, are infinite: Let $n \in \mathbb{N}$ and $i_{0}=0$. Put $M_{n}=\bigcup_{k=i_{n-1}+1}^{i_{n}} N_{k}$. Denote by $Y_{n}$ the closed linear span of 
$\left\{b_{j}: j \in M_{n}\right\}$. Since $r_{k}\left(b_{j}\right)=k$ for $j \in N_{k}, k \in \mathbb{N}$, then $r_{k}\left(b_{j}\right)=k$ for $j \in M_{n}$ and $k \geq i_{n}$. Hence $Y_{n}$ is normable, so it is isomorphic to $c_{0}$.

For any $\left(a_{n}\right) \in \prod_{n=1}^{\infty} Y_{n}$ the series $\sum_{n=1}^{\infty} a_{n}$ is convergent in $Y$. Indeed, let $k \in \mathbb{N}$. Since $r_{k}\left(b_{j}\right)=0$ for $j \in N_{k+1}$, then $r_{k}\left(b_{j}\right)=0$ for $j \in M_{n}, n \in \mathbb{N}$ with $i_{n-1} \geq k$. Hence $r_{k}\left(a_{n}\right)=0$ for any $n \in \mathbb{N}$ with $i_{n-1} \geq k$. Thus $\lim a_{n}=0$.

Let $T_{n}$ be the natural projection from $Y$ onto $Y_{n}, n \in \mathbb{N}$. Clearly, $y=\sum_{n=1}^{\infty} T_{n} y$ for any $y \in Y$. By the open mapping theorem ([5], Corollary 2.74), the continuous map $T: Y \rightarrow \prod_{n=1}^{\infty} Y_{n}, y \rightarrow\left(T_{n} y\right)$ is an isomorphism. Thus $Y$ is isomorphic to $c_{0}^{\mathbb{N}}$.

Case 4 For some $k_{0} \in \mathbb{N}$ the sets $W_{1}:=\bigcup_{k=1}^{k_{0}} N_{k}, W_{2}:=\bigcup_{k=k_{0}+1}^{\infty} N_{k}$ are infinite and the sets $N_{k}, k>k_{0}$, are finite: The closed linear span $Z_{1}$ of $\left\{b_{n}: n \in W_{1}\right\}$ is normable, since $r_{i}\left(b_{n}\right)=i$ for $i \geq k_{0}$ and $n \in W_{1}$. Thus $Z_{1}$ is isomorphic to $c_{0}$. The closed linear span $Z_{2}$ of $\left\{b_{n}: n \in W_{2}\right\}$ is of finite type, since $\left\{b_{n}: n \in \bigcup_{k=i+1}^{\infty} N_{k}\right\} \subset$ $Z_{2} \cap \operatorname{ker} r_{i}$ for any $i \geq k_{0}$. Thus $Z_{2}$ is isomorphic to $\mathbb{K}^{\mathbb{N}}$. Hence $Y$ is isomorphic to $c_{0} \times \mathbb{K}^{\mathbb{N}}$.

For $V^{\mathbb{N}}$ we have the following result (see the proof of Proposition 4).

Proposition 8 A Fréchet space $E$ with a basis is isomorphic to $V^{\mathbb{N}}$ if and only if any Fréchet space with a basis is isomorphic to a complemented subspace of E.

Remark 9 There exists a Fréchet space $X$ of countable type which is is not isomorphic to any complemented subspace of $V^{\mathbb{N}}$. Indeed, there is a nuclear Fréchet space $X$ with a continuous norm and without the bounded approximation property (see [9, Theorem 11]). Since any complemented subspace of a Fréchet space with a basis has the bounded approximation property, then $X$ is not isomorphic to any complemented subspace of $V^{\mathbb{N}}$.

For bases in nuclear Fréchet spaces we shall prove the following.

Theorem 10 There is no quasi-universal basis for the family $\mathcal{F}_{n}$ of all bases in nuclear Fréchet spaces or for the family $\mathcal{F}_{n c}$ of all bases in nuclear Fréchet spaces with continuous norms.

Proof Let $E$ be a nuclear Fréchet space with a basis $\left(x_{n}\right)$. Assume that $E$ is not of finite type. Then $\left(x_{n}\right)$ is 1-orthogonal with respect to a base $\left(q_{k}\right)$ in $\mathcal{P}(E)$ with $\operatorname{dim}\left(E / \operatorname{ker} q_{1}\right)=\infty$. Let $i \in \mathbb{N}$. Put $N_{i}=\left\{n \in \mathbb{N}: q_{i}\left(x_{n}\right)>0\right\}$. Clearly, the closed linear span $X_{i}$ of $\left\{x_{n}: n \in N_{i}\right\}$ is an infinite-dimensional nuclear Fréchet space and $\left(x_{n}\right)_{n \in N_{i}}$ is a basis in $X_{i}$ which is 1-orthogonal with respect to the base of norms $\left(q_{k} \mid X_{i}\right)_{k=i}^{\infty}$ in $\mathcal{P}\left(X_{i}\right)$. Therefore we have $\lim _{n \in N_{i}}\left[q_{i}\left(x_{n}\right) / q_{j}\left(x_{n}\right)\right]=0$ for some $j>i$. Thus we can construct inductively an increasing sequence $\left(i_{k}\right) \subset \mathbb{N}$ such that $\lim _{n \in N_{i_{k}}}\left[q_{i_{k}}\left(x_{n}\right) / q_{i_{k+1}}\left(x_{n}\right)\right]=0$ for any $k \in \mathbb{N}$. Put $p_{k}=q_{i_{k}}$ and $M_{k}=N_{i_{k}}$ for $k \in \mathbb{N}$. Let $a_{i, j}(n)=\left[p_{j}\left(x_{n}\right) / p_{i}\left(x_{n}\right)\right]$ for $k, i, j \in \mathbb{N}$ with $k \leq i<j$ and $n \in M_{k}$. Clearly, $\lim _{n \in M_{k}} a_{i, j}(n)=\infty$ for $k, i, j \in \mathbb{N}$ with $k \leq i<j$. Thus there exists an increasing sequence $\left(s_{n}\right) \subset \mathbb{N}$ such that for any $n>1$ we have

$$
\max _{1 \leq i<j \leq n} \max _{m \in M(n)} a_{i, j}(m)<\min _{1 \leq k \leq n} \min _{m \in M_{k}\left(s_{n}\right)} a_{k, k+1}(m)
$$


where $M(n)=\left\{m \in M_{1}: m \leq n\right\}$ and $M_{k}\left(s_{n}\right)=\left\{i \in M_{k}: i \geq s_{n}\right\}$ (we assume that $\max \varnothing=0)$. Let $t_{j}=\min \left\{n \in M_{1}: s_{n} \geq j\right\}$ for $j \in \mathbb{N}$. Put $b_{i, j}=p_{i}\left(x_{t_{j}}\right)$ for all $i, j \in \mathbb{N}$. Then $0<b_{i, j} \leq b_{i+1, j}$ for all $i, j \in \mathbb{N}$ and $\lim _{j}\left(b_{i, j} / b_{i+1, j}\right)=$ $\lim _{j} a_{i, i+1}^{-1}\left(t_{j}\right)=0$ for any $i \in \mathbb{N}$. Thus for $B=\left(b_{i, j}\right)$ the Köthe space $K(B)$ is nuclear. We shall show that the coordinate basis $\left(e_{n}\right)$ in $K(B)$ is not quasi-equivalent to any subbasis of $\left(x_{n}\right)$. Suppose, by contradiction, that $\left(e_{n}\right)$ is equivalent to $\left(\alpha_{n} x_{k_{\pi(n)}}\right)$ for some $\left(\alpha_{n}\right) \subset(\mathbb{K} \backslash\{0\})$, some increasing sequence $\left(k_{n}\right) \subset \mathbb{N}$ and some permutation $\pi$ of $\mathbb{N}$. Then there exists a linear homeomorphism $T: \operatorname{lin}\left(e_{n}\right) \rightarrow \operatorname{lin}\left(x_{k_{n}}\right)$ with $T\left(e_{n}\right)=$ $\alpha_{n} x_{k_{\pi(n)}}$ for any $n \in \mathbb{N}$. By the continuity of $T$ and $T^{-1}$ we obtain

$$
\forall m \in \mathbb{N} \exists u(m), v(m) \in \mathbb{N} \forall x \in \operatorname{lin}\left(e_{n}\right): p_{m}(T x) \leq r_{u(m)}(x) \leq p_{v(m)}(T x)
$$

where $\left(r_{k}\right)$ is the standard base of norms in $\mathcal{P}(K(B))$. Clearly, we can assume that $u(m)<u(m+1)$ for any $m \in \mathbb{N}$.

Then $\forall m, n \in \mathbb{N}: p_{m}\left(x_{k_{\pi(n)}}\right) \leq r_{u(m)}\left(\alpha_{n}^{-1} e_{n}\right) \leq p_{v(m)}\left(x_{k_{\pi(n)}}\right)$. Thus

$$
\forall m, n \in \mathbb{N}:\left[p_{m}\left(x_{k_{\pi(n)}}\right) / r_{u(m)}\left(e_{n}\right)\right] \leq\left|\alpha_{n}^{-1}\right| \leq\left[p_{v(m)}\left(x_{k_{\pi(n)}}\right) / r_{u(m)}\left(e_{n}\right)\right]
$$

Hence $\forall i, j, n \in \mathbb{N}:\left[p_{j}\left(x_{k_{\pi(n)}}\right) / r_{u(j)}\left(e_{n}\right)\right] \leq\left[p_{v(i)}\left(x_{k_{\pi(n)}}\right) / r_{u(i)}\left(e_{n}\right)\right]$.

Since $\forall n \in \mathbb{N}: p_{v(1)}\left(x_{k_{\pi(n)}}\right) \geq r_{u(1)}\left(\alpha_{n}^{-1} e_{n}\right)>0$, then $\left\{k_{\pi(n)}: n \in \mathbb{N}\right\} \subset M_{v(1)}$ and $\forall i, j, n \in \mathbb{N}:\left[r_{u(j)}\left(e_{n}\right) / r_{u(i)}\left(e_{n}\right)\right] \geq\left[p_{j}\left(x_{k_{\pi(n)}}\right) / p_{v(i)}\left(x_{k_{\pi(n)}}\right)\right]$. Thus we have

$$
\forall i, j, n \in \mathbb{N}: a_{u(i), u(j)}\left(t_{n}\right) \geq a_{v(i), j}\left(k_{\pi(n)}\right)
$$

Let $j=v(1)+1$ and $n \in M_{1}$ with $n>u(j)$. Since $\max \left\{k_{\pi(b)}: 1 \leq b \leq s_{n}\right\} \geq s_{n}$, there exists $d \in \mathbb{N}$ with $d \leq s_{n} \leq k_{\pi(d)}$. Hence $t_{d} \leq n$ and $k_{\pi(d)} \in M_{v(1)}\left(s_{n}\right)$. Thus by (*) we get $a_{u(1), u(j)}\left(t_{d}\right)<a_{v(1), j}\left(k_{\pi(d)}\right)$, contrary to $(* *)$.

It follows that the basis $\left(x_{n}\right)$ is not universal for $\mathcal{F}_{n}$ or $\mathcal{F}_{n c}$. This completes the proof, since any basis in a Fréchet space of finite type is not universal for $\mathcal{F}_{n}$.

\section{References}

[1] N. De Grande-De Kimpe, On the structure of locally $\mathbb{K}$-convex spaces with a Schauder basis. Indag. Math. 34(1972), 396-406.

[2] Won-archimedean Fréchet spaces generalizing spaces of analytic functions. Indag. Math. 44(1982), 423-439.

[3] N. De Grande-De Kimpe, J. Kạkol, C. Perez-Garcia and W. H. Schikhof, Orthogonal sequences in non-archimedean locally convex spaces. Indag. Math. N.S. 11(2000), 187-195.

[4] Orthogonal and Schauder bases in non-archimedean locally convex spaces. p-adic functional analysis. Ioannina 2000, 103-126, Lecture Notes in Pure and Appl. Math. 222, Marcel Dekker, New York, 2001.

[5] J. B. Prolla, Topics in functional analysis over valued division rings. North-Holland Math. Studies 77, North-Holland Publ. Co., Amsterdam, 1982.

[6] A. C. M. van Rooij, Non-archimedean functional analysis. Marcel Dekker, New York, 1978.

[7] W. H. Schikhof, Locally convex spaces over non-spherically complete valued fields. Bull. Soc. Math. Belg. 38(1986), 187-224. 
[8] W. Śliwa, Every infinite-dimensional non-archimedean Fréchet space has an orthogonal basic sequence. Indag. Math. N.S. (3)11(2000), 463-466.

[9] Examples of non-archimedean nuclear Fréchet spaces without a Schauder basis. Indag. Math. N.S. (4) 11(2000), 607-616.

Faculty of Mathematics and Computer Science

A. Mickiewicz University

ul. Umultowska 87

61-614 Poznań

Poland

e-mail: sliwa@amu.edu.pl 